\title{
UREA AND CREATININE LEVELS IN VAGINAL FLUID- A RELIABLE MARKER FOR PRELABOUR RUPTURE OF MEMBRANES
}

\author{
Kshama Kedar1, Jayant Patil2, Suvarna Nimkar ${ }^{3}$
}

${ }_{1}^{1}$ Associate Professor, Department of Obstetrics and Gynaecology, Indira Gandhi Government Medical College, Nagpur, Maharashtra. ${ }^{2}$ Resident, Department of Obstetrics and Gynaecology, Indira Gandhi Government Medical College, Nagpur, Maharashtra. ${ }^{3}$ Assistant Professor, Department of Obstetrics and Gynaecology, Indira Gandhi Government Medical College, Nagpur, Maharashtra.

\section{ABSTRACT}

\section{BACKGROUND}

Prelabour rupture of membranes is known to have adverse effect on pregnant women with unfavourable foetal outcome. Failure to identify membrane rupture can result in failure to implement obstetric measures, while false diagnosis can lead to inappropriate interventions. Different tests are described, but there has been no gold standard test for diagnosing PROM.

Thus, to find out the test which is rapid, bedside, inexpensive and accurate led to the study for detection of levels of creatinine and urea in the vaginal fluid for diagnosis of PROM.

\section{MATERIALS AND METHODS}

This is a prospective case control study done over a time period of 2 years in a tertiary care institute. Total 160 pregnant women were divided into study and control groups, each of 80 . Vaginal fluid washing was collected, urea and the creatinine estimation was done by (GLDH) Kinetic Method and Jaffe's Method respectively.

\section{RESULTS}

Creatinine at a threshold value of $0.668 \mathrm{mg} / \mathrm{dL}$ had a sensitivity, specificity, PPV, NPV and accuracy of 100\%, 91.25\%, 91.95\%, $100 \%$ and $95.62 \%$ respectively. In the diagnosis of PROM for a threshold value of $9.906 \mathrm{mg} / \mathrm{dL}$ for urea, the sensitivity, specificity, PPV, NPV and accuracy were $100 \%, 92.5 \%, 93.02 \%, 100 \%$ and $96.25 \%$ respectively in the detection of PROM.

\section{CONCLUSION}

We found that determination of urea and creatinine in vaginal fluid is a reliable marker for diagnosing PROM. This is a simple, practical, cost-effective and easily accessible test if incorporated routinely in the low resource setting. This test has the potential of becoming the gold standard test for the diagnosis of PROM.

\section{KEY WORDS}

Prelabour Rupture of Membranes, Vaginal Fluid Urea and Creatinine Levels, Diagnosis of PROM.

HOW TO CITE THIS ARTICLE: Kedar K, Patil J, Nimkar S. Urea and creatinine levels in vaginal fluid- a reliable marker for prelabour rupture of membranes. J. Evolution Med. Dent. Sci. 2018;7(20):2456-2459, DOI: 10.14260/jemds/2018/553

\section{BACKGROUND}

Of the many problems complicating pregnancy, one of utmost importance is the Premature Rupture of Membranes (PROM). Premature Rupture of Membranes (PROM) refers to the rupture of amniotic membranes prior to the onset of labour regardless of gestational age, while preterm premature rupture of membranes (PPROM) means rupture of amniotic membranes before 37 weeks of gestational age. ${ }^{1}$ It complicates $8 \%$ of term pregnancies ( $\geq 37$ weeks), while Preterm Premature Rupture of Membranes (PPROM) complicates $2-4 \%$ of singleton and $7-20 \%$ of twin pregnancies. On an average $20-40 \%$ of preterm births are a result of PPROM and it accounts for $18-20 \%$ of perinatal deaths in developed countries and much more in the developing countries. ${ }^{2}$

'Financial or Other Competing Interest': None.

Submission 06-04-2018, Peer Review 30-04-2018,

Acceptance 07-05-2018, Published 14-05-2018.

Corresponding Author:

Dr. Suvarna Nimkar,

Assistant Professor,

Department of Obstetrics and Gynaecology,

Indira Gandhi Government Medical College,

Nagpur, Maharashtra.

E-mail: suvarnanimkar@gmail.com

DOI: $10.14260 /$ jemds $/ 2018 / 553$
PROM increases the risk of chorioamnionitis upto $15 \%$ and foetal infection risk upto 5\%. Foetal infection risk increases upto $20 \%$ in those with chorioamnionitis. Also, the hospital stay of neonates with premature rupture of membranes increases by $20-25 \%$ with the cost of hospitalisation increasing upto $60 \%$. There is also a risk of $1 \%$ endometritis in term and post-term period. ${ }^{3}$

Latency from membrane rupture to delivery is brief and is inversely proportional to gestational age with $93 \%$ of cases with PPROM delivering in a week. ${ }^{4}$ With PROM thus there are increased risk of preterm deliveries, caesarean sections and low birth weight babies, neonatal complications like respiratory distress syndrome, necrotising enterocolitis, inter-ventricular haemorrhage, cerebral palsy, septicaemia, neonatal deaths and perinatal mortality. ${ }^{5}$ Serious complications in conservative management occurring early are retained placenta and postpartum haemorrhage (PPH) necessitating dilatation and curettage $(12 \%)$, maternal sepsis $(0.8 \%)$ and maternal death $(0.14 \%){ }^{6}$

Diagnosis is easy when clinical situation is obvious, but difficult when there is doubt as to whether PROM has occurred or not. It is vital to diagnose this condition accurately. Different tests have been tried, but until now there has been no gold standard test for diagnosing PROM. ${ }^{7}$ Traditionally, minimally invasive gold standard for diagnosis of PROM relies on clinician's ability to document three clinical signs on sterile speculum examination: 1) Visual pooling of 
clear fluid in the posterior fornix of the vagina or leakage of the fluid from the cervical os; 2) An alkaline $\mathrm{pH}$ of the cervicovaginal discharge, which is typically demonstrated by nitrazine paper and/or 3) Microscopic ferning of the cervicovaginal discharge. ${ }^{8}$

Failure to identify membrane rupture can result in failure to implement obstetric measures, while false diagnosis can lead to inappropriate interventions such as hospitalisation or labour induction. ${ }^{9}$

The need to identify markers in amniotic fluid for inexpensive, rapid, bedside and accurate detection of PROM led to the studies for detection of levels of Creatinine and Urea in the vaginal washing fluid, which initiates this study.

\section{Aims and Objectives}

1. To determine the diagnostic accuracy of vaginal washing fluid urea and creatinine levels in the detection of PROM.

2. To give suitable recommendations based on the study findings.

\section{MATERIALS AND METHODS}

This is a prospective case-control study conducted in the Department of Gynaecology and Obstetrics in collaboration with the Department of Biochemistry at a tertiary care hospital in central India over the period of 2 years. The study consisted of antenatal patients admitted to the labour room. These patients are divided into two groups namely-

\section{Group I- The Study (Cases) Group}

This group consisted of pregnant patients with the history of leaking per vagina and confirmed leak on per speculum and per vaginal examination.

\section{Group II- The Control Group}

This group consisted of healthy pregnant patients who did not either had the history of leaking per vagina or leak per speculum/ vaginal examination.

All the patients are between 28 weeks and 42 weeks of gestation. Total sample size of the study is 160 . The study is approved by the ethics committee.

\section{Inclusion Criteria}

Antenatal women with singleton pregnancy, gestational age between 28 - 42 weeks without any foetal congenital renal anomaly or severe medical illness.

\section{Exclusion Criteria}

PROM in patients having multiple pregnancies, congenital foetal anomaly, history of vaginal infection, intrauterine foetal demise, meconium stained liquor and women not willing to participate in the study.

\section{Study Methodology}

After obtaining consent from pregnant female detailed history regarding leaking per vaginum and its duration along with obstetric history, menstrual history, history of past illness, immunisation history etc. was taken. Then the general examination including per abdomen examination was done. This was followed by local examination. The sample was collected during the sterile speculum examination, which was done for the observation of pooling of the amniotic fluid. During the examination, the posterior fornix was irrigated by
5 cc of Normal Saline, then 3 cc of this washing fluid was aspirated back in the same syringe and this sample was transferred in a sterile plain bulb. After proper labelling, this sample was then sent for further processing in the biochemical laboratory immediately. In case of delay, these samples were preserved in the refrigerators at $4-8^{\circ} \mathrm{C}$. In the biochemistry laboratory, the samples were centrifuged at 50 revs/min for 10 minutes. Then the supernatant fluid was used for the estimation of urea and creatinine. The samples were processed in the ERBA XL-640 automated analyser. Urea and creatinine estimation were done by (GLDH) Kinetic Method and Jaffe's Method (Initial Rate) respectively.

\section{Statistical Analysis}

The data were entered into Microsoft Excel and was analysed using SPSS Version 20.0. The qualitative data was represented in the form of frequency and percentage. The quantitative data was represented in the form of mean and standard deviation. The association between two qualitative variables was calculated by using chi-square test and the difference between two means in both groups was calculated using unpaired t-test. A p-value less than 0.05 was considered as statistically significant.

\section{RESULTS}

Most of the participants in both cases and control groups belonged to 21 - 30 years $(80.0 \%$ in cases and $88.8 \%$ in controls). There is no statistical significant difference between the two groups with regards to maternal age. Most of the participants in case group were primigravida (43.7\%), while in the control group most of the participants were of second gravida status $(45.0 \%)$. Both the groups are comparable with regards to gravida status. As per the LMP, $23.8 \%$ participants were preterm in the case group compared to $13.8 \%$ participants in the control group and as per the earliest ultrasound scan $26.3 \%$ participants were preterm in the case group compared to $12.5 \%$ participants of the control group. There was no statistically significant difference between the two groups with regards to GA as per LMP and scan.

\begin{tabular}{|c|c|c|c|c|c|}
\hline \multirow{3}{*}{$\begin{array}{l}\text { Urea Conc. } \\
\mathrm{mg} / \mathrm{dL}\end{array}$} & Group & $\mathbf{N}$ & Mean & $\begin{array}{l}\text { Standard } \\
\text { Deviation }\end{array}$ & P value \\
\hline & Case & 80 & 19.48 & 4.88 & \multirow{2}{*}{0.0001} \\
\hline & Control & 80 & 5.97 & 3.01 & \\
\hline \multirow{3}{*}{$\begin{array}{l}\text { Creatinine } \\
\text { Conc. } \\
(\mathrm{mg} / \mathrm{dL})\end{array}$} & Group & $\mathrm{N}$ & Mean & $\begin{array}{l}\text { Standard } \\
\text { deviation }\end{array}$ & $P$ value \\
\hline & Case & 80 & 1.22 & 0.28 & \multirow{2}{*}{0.0001} \\
\hline & Control & 80 & 0.36 & 0.26 & \\
\hline
\end{tabular}

Table 1. Comparison of Concentration of Urea and Creatinine in both the Groups

\begin{tabular}{|c|c|c|}
\hline \multirow[b]{2}{*}{ Urea Threshold $=9.906 \mathrm{mg} / \mathrm{dL}$} & \multicolumn{2}{|c|}{ PROM } \\
\hline & $\begin{array}{l}\text { Case } \\
\text { Group }\end{array}$ & $\begin{array}{l}\text { Control } \\
\text { Group }\end{array}$ \\
\hline Positive $(\geq 9.906 \mathrm{mg} / \mathrm{dL})$ & $80(\mathrm{TP})$ & $6(\mathrm{FP})$ \\
\hline Negative $(<9.906 \mathrm{mg} / \mathrm{dL})$ & $0(\mathrm{FN})$ & $74(\mathrm{TN})$ \\
\hline Creatinine Threshold $=0.668 \mathrm{mg} / \mathrm{dL}$ & $\begin{array}{l}\text { Case } \\
\text { Group }\end{array}$ & $\begin{array}{l}\text { Control } \\
\text { Group }\end{array}$ \\
\hline Positive (> $0.668 \mathrm{mg} / \mathrm{dL})$ & 80 (TP) & $7(\mathrm{FP})$ \\
\hline Negative $(<0.668 \mathrm{mg} / \mathrm{dL})$ & $0(\mathrm{FN})$ & $73(\mathrm{TN})$ \\
\hline $\begin{array}{l}\text { Table 2. Calculation of the Thres } \\
\text { Creatinine in the Vagina }\end{array}$ & Value o & Jrea and \\
\hline
\end{tabular}


(TP- True Positive, FP- False Positive, TN- True Negative, FN- False Negative).

\section{Calculation of the Threshold Value of Urea in the Vaginal Washing Fluid}

Standard Deviation was calculated as 4.88 and $95 \%$ confidence interval is given by $1.96 \mathrm{SD}$ below the mean value. Threshold value of urea= Mean $-1.96 \mathrm{SD}=9.906 \mathrm{mg} / \mathrm{dL}$ and any value above this indicated PROM.

\section{Calculation of the Threshold Value of Creatinine in the Vaginal Washing Fluid}

The standard deviation was calculated as 0.283 and $95 \%$ confidence interval is given by the value of $1.96 \mathrm{SD}=0.555$. Threshold value of creatinine $=$ Mean $-1.96 \mathrm{SD}=0.668 \mathrm{mg} / \mathrm{dL}$ and any value above this indicated PROM.

\begin{tabular}{|c|c|c|c|}
\hline \multirow{2}{*}{ Birth Weight } & \multicolumn{2}{|c|}{ Group } & \multirow{2}{*}{ Total } \\
\cline { 2 - 4 } & Case (n= 80) & Control (n= 80) & \\
\hline \multirow{2}{*}{ Low birth weight } & 32 & 25 & 57 \\
\cline { 2 - 4 } & $56.1 \%$ & $43.9 \%$ & $100.0 \%$ \\
\hline \multirow{2}{*}{ Normal birth weight } & 48 & 55 & 103 \\
\cline { 2 - 4 } & $46.6 \%$ & $53.4 \%$ & $100.0 \%$ \\
\hline Chi-square $=1.335, \mathrm{df}=1, \mathrm{p}-\mathrm{v}$-value $=0.248$ not significant \\
\hline \multicolumn{3}{|c|}{ Table 3. Association of Birth Weight between both the } \\
Groups \\
\hline
\end{tabular}

\begin{tabular}{|c|c|c|c|c|c|}
\hline \multicolumn{2}{|c|}{ Type of Delivery } & Cases & Controls & Total & Significance \\
\hline PTND & No. & 8 & 10 & 18 & \multirow{6}{*}{$\begin{array}{c}\text { Chi- } \\
\text { square }=12.4 \\
8, \mathrm{df}=1, \\
\mathrm{p}=0.0004 \\
\text { significant }\end{array}$} \\
\hline & $\%$ & $10 \%$ & $12.50 \%$ & $11.25 \%$ & \\
\hline \multirow[t]{2}{*}{ FTND } & No. & 28 & 48 & 76 & \\
\hline & $\%$ & $35 \%$ & $60 \%$ & $47.50 \%$ & \\
\hline \multirow[t]{2}{*}{$\begin{array}{c}\text { Total } \\
\text { Vaginal } \\
\text { Deliveries }\end{array}$} & No. & 36 & 58 & 94 & \\
\hline & $\%$ & $45 \%$ & $72.50 \%$ & $58.75 \%$ & \\
\hline \multirow[t]{2}{*}{$\begin{array}{l}\text { Preterm } \\
\text { LSCS }\end{array}$} & No. & 9 & 1 & 10 & \multirow{6}{*}{$\begin{array}{c}\text { Chi } \\
\text { square }=12.4 \\
8, d f=1 \\
p=0.0004 \\
\text { significant }\end{array}$} \\
\hline & $\%$ & $11.25 \%$ & $1.25 \%$ & $6.25 \%$ & \\
\hline \multirow[t]{2}{*}{ Term LSCS } & No. & 35 & 21 & 56 & \\
\hline & $\%$ & $43.75 \%$ & $26.25 \%$ & $35 \%$ & \\
\hline \multirow[t]{2}{*}{ Total LSCS } & No. & 44 & 22 & 66 & \\
\hline & $\%$ & $55 \%$ & $27.50 \%$ & $41.25 \%$ & \\
\hline
\end{tabular}

\section{DISCUSSION}

In the current study it was observed that the mean age of case group was $24.95 \pm 3.78$ years, while that of the control group was $24.96 \pm 3.23$ years. The $p$-value being $>0.05$ concludes no statistically significant difference with regards to maternal age in the two groups.

These results are identical with the study results of Tavana $\mathrm{Z}$ et al (2010),10 who observed the mean ages to be $25.2 \pm 5.7,25.4 \pm 5.6$ and $26.7 \pm 5.5$ years in the confirmed PROM, suspected PROM and the healthy control group respectively. Similarly, Kariman N et al (2013) ${ }^{8}$ in their study also found the mean ages to be $26.25 \pm 5.40,25.46 \pm 6.0$ and $25.54 \pm 4.69$ in the confirmed PROM, suspected PROM and the healthy control group respectively and Ghasemi M et al (2016) ${ }^{11}$ found the mean ages of the study participants to be $25.0 \pm 5.6$ and $25.8 \pm 5.5$ years in the confirmed PROM and the control group respectively with no statistically significant difference in between the two groups.

The observed mean gravida status of the case group and the control group was $1.91 \pm 0.83$ and $2.04 \pm 0.83$ respectively and the $p$-value was observed to be $>0.05$. Thus, there was no statistically significant difference in the two groups and the two groups under study were identical. Also, it was noted that most of the participants in the case group were primigravida (43.7\%), while in the control group most of the participants were of second gravida status. Similar results were found in the study of Tavana $\mathrm{Z}$ et al (2010), ${ }^{10}$ Kariman N et al (2013) ${ }^{8}$ and Begum J et al (2017).12

The mean gestational age in the case group was $37.48 \pm$ 2.03 weeks and in the control group was $38.28 \pm 1.68$ weeks as per calculations from the dates of last menstrual period. Thus, it was observed that there was no statistically significant difference between the groups.

The distribution of study participants as per gestational age as per the earliest scan shows the mean gestational age of $37.16 \pm 2.25$ weeks and $38.13 \pm 1.67$ weeks in the case and the control group respectively. Thus, it was seen that no statistically significant difference was present in the two study groups. Thus, the mean gestational ages in both the study groups are identical. Conversely, we can say that there was no statistically significant difference in the case and the control group with regards to gestational age. Similar results were found by Kariman N et al (2013), 8 Tigli A et al (2014) ${ }^{13}$ and Begum J et al (2017) ${ }^{12}$ in their studies.

In the study by Tavana $\mathrm{Z}$ et al (2010), ${ }^{10}$ the mean gestational ages are lower than those observed in our study. But similar to our study, there is no statistically significant difference with regards to the gestational age between the study groups.

In present study, the mean concentration of urea was found to be $19.48 \pm 4.88$ and $5.97 \pm 3.01 \mathrm{mg} / \mathrm{dL}$ in the case and the control group respectively. This shows a statistically significant difference in the value of urea in the two groups. The threshold value of $9.906 \mathrm{mg} / \mathrm{dL}$ was worked out. It showed a sensitivity of $100 \%$, specificity of $92.5 \%$, PPV of $93.02 \%$, NPV of $100 \%$ and an accuracy of $96.25 \%$ in diagnosing PROM.

Also, it was observed that the mean concentration of creatinine was $1.22 \pm 0.28 \mathrm{mg} / \mathrm{dL}$ in the case group and 0.36 $\pm 0.26 \mathrm{mg} / \mathrm{dL}$ in the control group. Thus, there is a statistically significant difference in the two groups regarding the creatinine concentration. The cut-off value for creatinine worked out to be $0.668 \mathrm{mg} / \mathrm{dL}$ which showed $100 \%$ sensitivity, $91.25 \%$ specificity, PPV of $91.95 \%$, NPV of $100 \%$ and accuracy of $95.62 \%$ for the detection of PROM.

The difference in the values of urea and creatinine concentration in the different studies might be due to the different inclusion criteria for the study participants, a difference in the gestational ages, different dilutions used in sample collection and the different biochemical procedures used for estimation of urea and creatinine.

In this study, we observed that $56.1 \%$ of the babies delivered in the confirmed case group were low birth weight (LBW) compared to $43.9 \%$ in the control group. Thus, more LBW babies were delivered in the case group as compared to the control group, but this difference was not statistically significant. 
It is observed that preterm vaginal deliveries occurred in $10 \%$ of participants in the case group as compared to $12.5 \%$ participants in the control group, whereas full-term vaginal deliveries were seen in 35\% participants of the case group as compared to $60 \%$ participants in the control group. Preterm lower segment caesarean section (LSCS) was performed in $11.25 \%$ of the participants of the case group compared to $1.25 \%$ participants of the control group, whereas $43.75 \%$ of study subjects in the case group had undergone term LSCS to terminate the pregnancy compared to $26.25 \%$ study subjects in the control group. Thus, we observed that 55\% participants in case group underwent LSCS compared to $27.5 \%$ of the control group. This is a statistically significant difference in the two study groups with regards to the type of delivery.

The current study is contradictory to the study by Effat DM et al (2012),14 who found no statistically significant difference in the type of delivery between the case, suspect and the control group. In their study vaginal deliveries were conducted in $72 \%, 76 \%$ and $60 \%$ of participants of the case, the suspect and the control group respectively.

Corresponding $28 \%, 24 \%$ and $40 \%$ participants in the three groups underwent LSCS for the termination of pregnancy.

\section{CONCLUSION}

We found that determination of urea and creatinine is a reliable method for diagnosis of PROM, thus can be used as an adjunctive test in confirming PROM. Both the urea and the creatinine have equal sensitivity and specificity in diagnosing PROM. This test is simple, practical, cost-effective and easily accessible test if incorporated routinely for the detection of PROM in the low resource community. Overall, this test has the potential of becoming the gold standard test for the diagnosis of PROM.

\section{REFERENCES}

[1] DeFranco E, Atkins K, Heyl PS. Preterm labor, premature rupture of membranes and cervical insufficiency. Evans, Arthur T, eds. Manual of Obstetrics. $7^{\text {th }}$ edn. Lippincott Williams \& Wilkins 2007;8:135-50.

[2] Spinillo A, Montanari L, Sanpaolo P, et al. Fetal growth and infant neurodevelopmental outcome after preterm premature rupture of membranes. Obstet Gynecol 2004;103(6):1286-93.

[3] Benedetto C, Tibaldi C, Marozio L, et al. Cervicovaginal infections during pregnancy: epidemiological and microbiological aspects. J Matern Neonatal Med 2004;16(Suppl 2):9-12.
[4] Mercer B, Arheart KL. Antimicrobial therapy in expectant management of preterm premature rupture of membranes. Lancet 1995;346(8985):1271-9.

[5] ACOG Committee on Practice Bulletins-Obstetrics. ACOG Practice Bulletin No. 80: premature rupture of membranes. Clinical management guidelines for obstetrician-gynecologists. Obstet Gynecol 2007;109(4):1007-19.

[6] Mercer BM, Moretti ML, Prevost RR, et al. Erythromycin therapy in preterm premature rupture of membranes: a prospective, randomized trial of 220 patients. Am J Obstet Gynecol 1992;166(3):794-802.

[7] Kafali H, Oksuzler C. Vaginal fluid Urea \& Creatinine in diagnosis of premature rupture of membranes. Arch Gynaecol Obstet 2007;275(3):157-60.

[8] Kariman N, Afrakhte M, Hedayati M, et al. Diagnosis of premature rupture of membranes by assessment of urea and creatinine in vaginal washing fluid. Iran Journal of Reproductive Medicine 2013;11(2):93-100.

[9] Medina TM, Hill DA. Preterm premature rupture of membranes: diagnosis and management. Am Fam Physician 2006;73(4):659-64.

[10] Tavana Z, Hamedi B, Zolghadri J, et al. The evaluation of diagnostic role of vaginal fluid urea, creatinine and I-HCG level for detection of premature rupture of membrane. The Internet Journal of Gynecology and Obstetrics 2010;15(1):1-6.

[11] Ghasemi M, Jaami R, Alleyassin A, et al. The value of urea, creatinine, prolactin and beta sub-unit of human chorionic gonadotropin of vaginal fluid in the diagnosis of premature preterm rupture of membranes in pregnancy. Turk J Obstet Gynecol 2016;13(2):62-6.

[12] Begum J, Samal SK, Ghose S, et al. Vaginal fluid urea and creatinine in the diagnosis of premature rupture of membranes in resource limited community settings. J Fam Reprod Health 2017;11(1):43-9.

[13] Tigli A, Kurt S, Kopuz A. The roles of beta-human chorionic gonadotropin, creatinine and urea in vaginal washing fluid in the diagnosis of premature rupture of membranes. Perinatal Journal 2014;22(3):133-7.

[14] Effat DM, Elkafrawy MAS, Elbagory E. The role of vaginal fluid creatinine versus human chorionic gonadotropin in diagnosis of premature rupture of membranes. AAMJ 2012;10(3):299-316. 